Editora Omnis Scientia

ANAIS DA I JORNADA NORTE-NORDESTE DE ENGENHARIAS (ONLINE)

Volume 1

$1^{\text {a }}$ Edição

TRIUNFO - PE 


\section{Coordenador Científico}

Alex Aguiar da Silva

\section{Coordenador de Publicação}

Daniel Luís Viana Cruz

\section{Coordenadora do Evento}

Andréa Telino Gomes

\section{Organizadores}

Academics - Eventos acadêmicos online

Editora Omnis Scientia

Andréa Telino Gomes

Daniel Luís Viana Cruz

Alex Aguiar da Silva

\section{Palestrantes}

Alex Aguiar da Silva

André Búrigo Leite

Cecília Aguiar da Silva

Eugênio Bastos da Costa

Jorge Recarte Henriquez Guerrero

Renan Ferreira da Rocha

\section{Avaliadores}

Alex Aguiar da Silva

Helio Cardoso Martim

Péricles Felipe Bastos Gomes

\section{Imagem de Capa}

Freepik

\section{Revisão}

Os autores

\section{(9) $\mathbb{\oplus \Theta \Theta}$}

Este trabalho está licenciado com uma Licença Creative Commons - Atribuição-NãoComercialSemDerivações 4.0 Internacional.

O conteúdo abordado nos artigos, seus dados em sua forma, correção e confiabilidade são de responsabilidade exclusiva dos autores. 


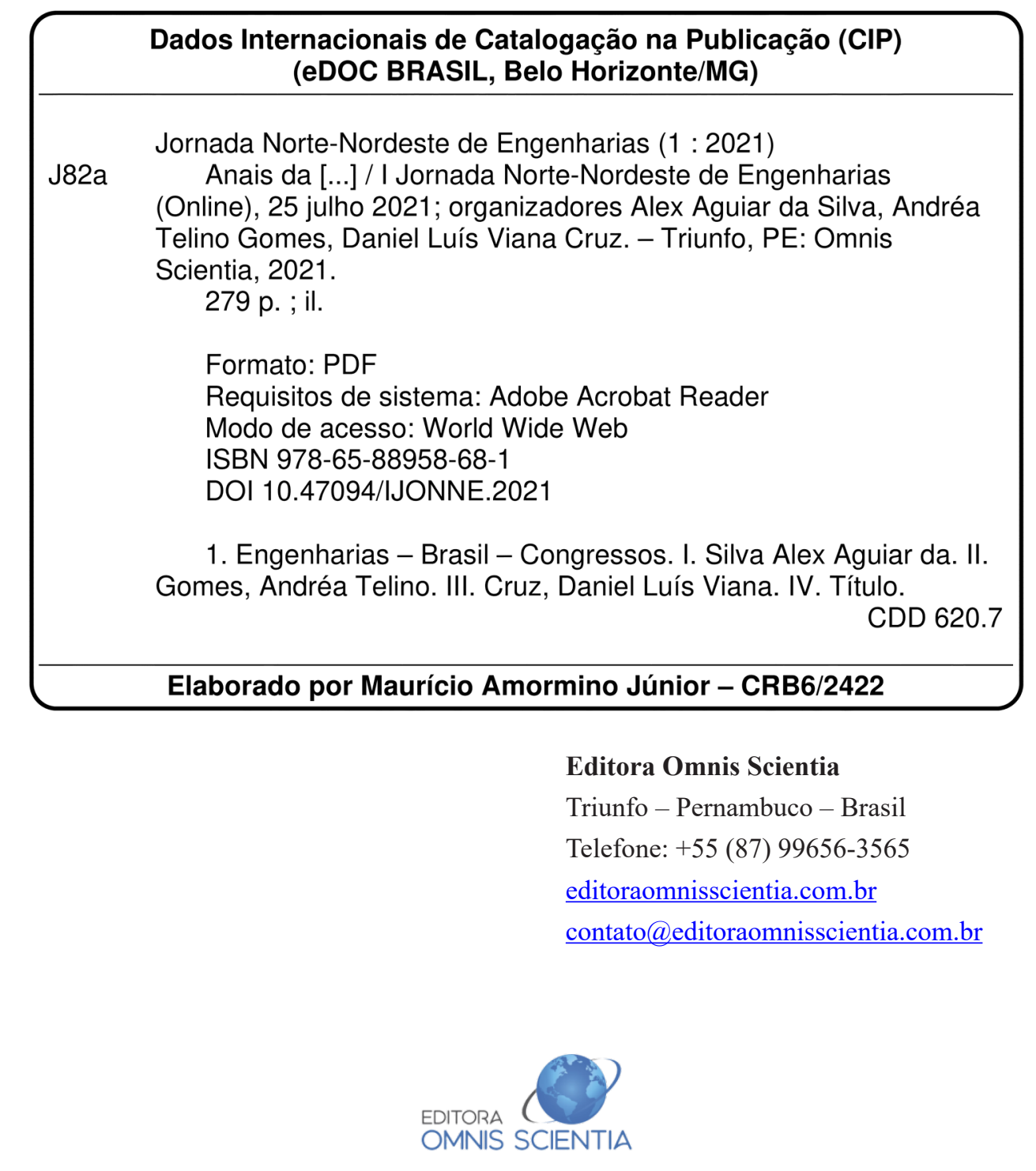




\section{EDITORIAL}

A grande área das engenharias abrangente uma série de ramos específicos, cada qual com determinados campos de aplicação e tipos de tecnologias, conjugando conhecimentos especializados no sentido de viabilizar as utilidades, tendo em conta a sociedade, a técnica, a economia e o meio ambiente. Deste modo, as engenharias aplicam o conhecimento científico, econômico, social e prático, para criar, desenhar, construir, manter e melhorar estruturas, máquinas, aparelhos, sistemas, materiais e processos. Assim, se adquire e se aplicam os conhecimentos matemáticos e técnicos na invenção, aperfeiçoamento e implementação de utilidades que realizem uma função ou objetivo.

A I Jornada Norte-Nordeste de Engenharias (online) I JONNE, objetivou reunir e integrar as engenharias para promover e apoiar esta área do conhecimento por meio de palestras, submissões de trabalhos na modalidade de resumos expandidos e exposição dos resumos aprovados.

Os participantes receberam certificados de participação de 20 horas e foi concedido menção honrosa para os três melhores trabalhos. Seguem os títulos dos resumos que foram premiados:

Modalidade resumo expandido

387210 - DIMENSIONAMENTO DE TROCADOR DE CALOR A PLACAS PARA PASTEURIZAÇÃO DE LEITE PELO PROCESSO HTST

387222 - DESENVOLVIMENTO DE UM DISPENSER DE ÁLCOOL GEL AUTOMÁTICO PARA MONITORAMENTO DA COMPLACÊNCIA DE MÃOS

387225 - REDE NEURAL ARTIFICIAL PARA VISCOSIDADE E CONDUTIVIDADE TÉRMICA DO FLUIDO REFRIGERANTE R32

A equipe organizadora da I JONNE agradece a todos os participantes, palestrantes e avaliadores pela participação e colaboração no congresso. 


\section{SUMÁRIO}

\section{ENGENHARIA CIVIL}

ANÁLISE DO USO DE RESÍDUO DE BRITAGEM DAS ROCHAS EM CONCRETO AUTOADENSÁVEL: UMA REVISÃO DA LITERATURA.....................................................12

ESTRATÉGIAS PARA A PREVENÇÃO DE ACIDENTES NA CONSTRUÇÃO CIVIL: UMA REVISÃO SISTEMÁTICA DA LITERATURA.

RESISTÊNCIA À COMPRESSÃO DE MISTURAS DE SOLO-CIMENTO COM NANOSSÍLICA E SÍLICA ATIVA.

PATOLOGIAS EM OBRAS PARALISADAS: O CASO DO ESTÁDIO COLOSSO DO TAPAJÓS NA CIDADE DE SANTARÉM - PA.

DIFERENÇA NO CUSTO DE MÃO DE OBRA EM FABRICAÇÃO DE LAJES PRÉ- MOLDADAS EM AMBITO NACIONAL

ESTUDO COMPARATIVO DE FUNDAÇÕES PARA UM EDIFÍCIO MODELO EM JOÃO PESSOA/PB: SAPATA X ESTACA HÉLICE CONTÍNUA.

MANIFESTAÇÕES PATOLÓGICAS EM EDIFICAÇÕES COM PAREDES DE CONCRETO ARMADO: ESTUDO DE CASO EM OBRA NA CIDADE DE JOÃO PESSOA - PB.....

REUTILIZAÇÃO DO PÓ RESIDUAL DE MARMORARIA PARA SUBSTITUIÇÃO DO AGREGADO MIÚDO NO CONCRETO.

SUBSIDÊNCIA OCORRIDA NOS BAIRROS PINHEIRO, BEBEDOURO E MUTANGE EM MACEIÓ - AL: UMA REVISÃO BIBLIOGRÁFICA.

CARACTERIZAÇÃO FÍSICA DAS VARIAÇÕES DIMENSIONAIS DO BAMBU GUADUA WEBERBAUERI DE RIO BRANCO - AC.

ESTUDO DA OCORRÊNCIA DE SOLOS COLAPSÍVEIS NA REGIÃO NORDESTE DO BRASIL.

OS EFEITOS DO USO DE POÇOS ARTESIANOS NO PROCESSO DE SALINIZAÇÃO COSTEIRA NA CIDADE DE SÃO LUÍS: UM LEVANTAMENTO BIBLIOGRAFICO.

TEORIADOS SÓLIDOS CELULARES EMPREGADOS EM REFORÇOS PARAACONSTRUÇÃO CIVIL

AVALIAÇÃO DO EXTRATO DE BOLDO E AROEIRA COMO INIBIDOR VEGETAL DE BAIXO CUSTO......

ANÁLISE DE DOSAGEM DE MATRIZ CIMENTÍCIA COM FIBRAS DE POLIPROPILENO: REVISÃO DA LITERATURA 
ANÁLISE DA RETENÇÃO DE CLORETOS EM ARGAMASSAS COM RESÍDUOS DE CELULOSE.

\section{ENGENHARIA DE MATERIAIS E METALÚRGICA}

ANÁLISE DA DEGRADAÇÃO FERRÍTICA DO AÇO INOXIDÁVEL DUPLEX SAF 2205 SUBMETIDO A ENVELHECIMENTO ISOTÉRMICO.

ANÁLISE DE INCLUSÕES E QUANTIFICAÇÃO DE POROSIDADE DO BRONZE AO ESTANHO EM ESTRUTURA BRUTA DE FUSÃO. 100

SÍNTESE RÁPIDA DE NANOFIOS DE NIOBATO DE SÓDIO. 105

AVALIAÇÃODAINCORPORAÇÃODACAREPADELAMINAÇÃOCOMOPARTEDAMATÉRIAPRIMA NA PRODUÇÃO DE SÍNTER DE FINOS DO MINÉRIO DE FERRO. 110

ESTUDO PROSPECTIVO DO PETRÓLEO APLICADO EM NANOMATERIAL CERÂMICO...... 115

ESTUDO PROSPECTIVO DE NANOMATERIAL CERÂMICO APLICADO EM SAÚDE MÉDICA E DENTÁRIA.

ESTUDO PROSPECTIVO DE MAGNETITA NANOMÉTRICA COMO MATERIAL FERROMAGNETICO.

ESTUDO PROSPECTIVO DE CERÂMICA AVANÇADA UTILIZANDO DOPAGEM......

BUSCA DE ANTERIORIDADE DE CERÂMICA APLICADA NA REMOÇÃO DE CONTAMINANTE..... .131

APLICAÇÕES DE $\mathrm{MoO}_{3}$ OBTIVO ATRAVÉS DE PRECIPITAÇÃO. 136

REVISÃO BIBLIOGRÁFICA SOBRE TUNGSTATO DE BÁRIO EM MEIO AQUOSO. 140

ESTUDOS DA DIFUSÃO DE CARBONO NOS AÇOS SAE 1020 E 1045 SUBMETIDOS À TRATAMENTO TERMOQUÍMICO DE CEMENTAÇÃO SÓLIDA. 145

\section{ENGENHARIA DO PRODUTO}

DESENVOLVIMENTO DE UM DISPENSER DE ÁLCOOL GEL AUTOMÁTICO PARA MONITORAMENTO DA COMPLACÊNCIA DE MÃOS. 150

\section{ENGENHARIA ELÉTRICA}

ENERGIA EÓLICA NO CEARÁ .156

\section{ENGENHARIA HIDRÁULICA}

ESTUDO DE MÉTODOS ANALÍTICOS PARA DETERMINAÇÃO DE PERDA DE CARGA EM TUBULAÇÕES DE PVC UTILIZADAS EM IRRIGAÇÕES. 162 
DESENVOLVIMENTO DE PRÁTICAS LABORATORIAIS APLICANDO OS CONCEITOS DE FENÔMENOS DE TRANSPORTES. 168

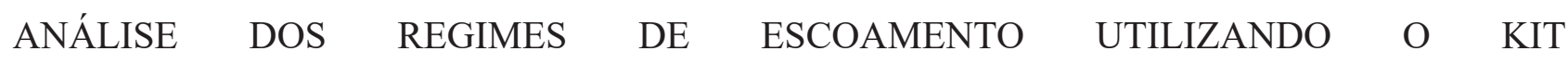
AQUALIBRIUM...

\section{ENGENHARIA MECÂNICA}

AVANÇO NO EMPREGO DE GABARITOS DE SOLDAGEM NO PROCESSO DE FABRICAÇÃO DE CHASSIS FORMULA SAE..

ANÁLISE DA DISTRIBUIÇÃO DE TEMPERATURA NOS PNEUS DE UM VEÍCULO FORMULA SAE PARA DIFERENTES CARGAS VERTICAIS. 184

\section{ENGENHARIA QUÍMICA}

UTILIZAÇÃO DE OXIDAÇÃO AVANÇADA PARA O TRATAMENTO DE EFLUENTES INDUSTRIAIS: UMA REVISÃO DA LITERATURA..

ANÁLISE DOS PRINCIPAIS PROCESSOS DE PRODUÇÃO DE BIODIESEL: UMA REVISÃO DE LITERATURA.

A QUÍMICA DOS AGROTÓXICOS USADOS EM AGRICULTURA: UMA REVISÃO DA LITERATURA. 201

AVALIAÇÃO CINÉTICA DA REAÇÃO DE DEGRADAÇÃO DE DIURON PRESENTE EM ÁGUAS SUPERFICIAIS.

ESTUDO DE CASO DO TRATAMENTO DE GÁS ÁCIDO COM COLUNA DE ABSORÇÃO DE LEITO RECHEADO.

ELABORAÇÃO DA CURVA DE CALIBRAÇÃO PARA ACRILAMIDA PELO MÉTODO DE CROMATOGRAFIA LÍQUIDA DE ALTA EFICIÊNCIA.

DETERMINAÇÃODECONSTANTESCINÉTICASPELOS MÉTODOSINTEGRALEDERUNGEKUTTA PARA REAÇÃO DE OXIDAÇÃO DO ANTRACENO.

DIMENSIONAMENTO DE TROCADOR DE CALOR A PLACAS PARA PASTEURIZAÇÃO DE LEITE PELO PROCESSO HTST.. 229

REDE NEURAL ARTIFICIAL PARA VISCOSIDADE E CONDUTIVIDADE TÉRMICA DO FLUIDO REFRIGERANTE R32.

TRANSFORMAÇÃO DO POLITEREFTALATO DE ETILENO RECICLADO EM FIBRAS TÊXTEIS DE POLIÉSTER. .239

\section{ENGENHARIA SANITÁRIA}

O USO DA ENERGIA SOLAR EM HOSPITAIS EM TEMPOS DE PANDEMIA 
ESTUDO DE VARIÁVEIS QUE POSSAM INFLUENCIAR NO CONSUMO DE ÁGUA DA CIDADE DE BAIXO GUANDÚ-ES........

\section{OUTRAS}

SISTEMAAUTOMÁTICO DE IRRIGAÇÃO. .260

A FRUTA DO MILAGRE - SYNSEPALUM DULCIFICUM.. 266 FOSSAS DE EVAPOTRANSPIRAÇÃO: UMA SOLUÇÃO SUSTENTÁVEL PARA OS DEFICITS NO SANEAMENTO EM ÁREAS CARENTES. .271

PROJETO DE ROBÓTICA SOCIAL E EDUCACIONAL .276 


\title{
ANÁLISE DA DEGRADAÇÃO FERRÍTICA DO AÇO INOXIDÁVEL DUPLEX SAF 2205 SUBMETIDO A ENVELHECIMENTO ISOTÉRMICO
}

\author{
Renan Abdalla Bandeira'; Camila Ferreira da Silva²; Flavio Ferreira ${ }^{3}$ \\ 33 \\ Engenheiro Metalúrgico, Universidade Federal Fluminense (UFF), Volta Redonda, RJ. \\ MSc. Engenharia Metalúrgica, Universidade Federal Fluminense (UFF), Volta Redonda, RJ. \\ Prof. DSc. Engenharia Metalúrgica, Universidade Federal Fluminense (UFF), Volta \\ Redonda, RJ.
}

DOI: 10.47094/IJONNE.2021.7

\begin{abstract}
RESUMO
Este trabalho traz a aplicação da técnica de ferritoscopia para investigar a dinâmica de consumo da fase ferrítica em prol da nucleação da fase sigma em um aço inoxidável duplex SAF 2205. Efetuouse o tratamento térmico de envelhecimento segundo intervalos variados na isoterma de $820^{\circ} \mathrm{C}$. A dureza do aço foi averiguada pelo método de ensaio Rockwell C. Ocorreu uma significativa redução no teor de ferrita, o que associado ao aumento da dureza sugere a formação da fase sigma no aço.
\end{abstract}

PALAVRAS-CHAVE: Aço inoxidável. SAF 2205. Ferritoscopia. Fase sigma.

ÁREA TEMÁTICA: Engenharia de Materiais e Metalúrgica

\section{INTRODUÇÃO}

Os aços inoxidáveis duplex estão sendo cada vez mais empregados na indústria. Possuem basicamente uma matriz ferrítico-austenítica, sendo as frações volumétricas destas duas fases semelhantes (OLIVEIRA, 2015). Devido a esta estrutura, são chamados aços bifásicos (SANTOS, 2008). Possuem elevada resistência a corrosão onde particularmente o aço SAF 2205, objeto deste estudo, supera a dos aços austeníticos (MENDONÇA, 2013). Suas maiores aplicações incluem a indústria petroquímica, náutica e nuclear devido sua resistência à corrosão por fadiga e excelentes propriedades mecânicas (WANG et al., 2019).

Quando expostos a alguns fatores tais como tratamento térmico ou elevadas temperaturas de trabalho, fases secundárias podem precipitar. A exposição prolongada a elevadas temperaturas (600 a $1000^{\circ} \mathrm{C}$ ) pode levar a transformação da ferrita em fase sigma (PADILHA, 2007). Em alguns aços como o SAF 2205, durante o processo de envelhecimento no intervalo de temperatura entre $800^{\circ} \mathrm{C}$ a $900^{\circ} \mathrm{C}$ a cinética de consumo da ferrita e precipitação da fase sigma se mostra mais acelerada 
(TAVARES et al., 2010). Esta é uma fase que causa a diminuição da tenacidade do aço devido a modificação na sua microestrutura bifásica, consumindo a priori a fase mais dúctil e macia, ferrita. A presença da fase sigma, de grande dureza, implica no aumento na dureza do material.

Dentre as várias técnicas para avaliação da fração ferrítica, a ferritoscopia se destaca pela adaptabilidade em ambientes industriais devido sua portabilidade e natureza não destrutiva do ensaio (CAMERINI et al., 2018).

\section{METODOLOGIA}

O material de estudo utilizado foi uma chapa de aço inoxidável duplex SAE 2205, laminada a quente, cortada em 5 amostras de dimensões $20 \mathrm{~mm}$ x $20 \mathrm{~mm}$ x $10 \mathrm{~mm}$. Cada uma das amostras foi levada ao forno Mufla Quimis Q318M24 em intervalos de tempo variando de 10 a 300 minutos, onde foram submetidas a uma temperatura isoterma de $820^{\circ} \mathrm{C}$, conforme Tabela 1 . O tratamento térmico foi seguido de resfriamento a temperatura ambiente.

Tabela 1: Especificações do tratamento térmico.

\begin{tabular}{ccc}
\hline Amostra & Tempo $[\mathrm{min}]$ & Temperatura $\left[{ }^{\circ} \mathrm{C}\right]$ \\
\hline A1 & 10 & 820 \\
\hline A2 & 30 & 820 \\
\hline A3 & 90 & 820 \\
\hline A4 & 120 & 820 \\
\hline A5 & 300 & 820 \\
\hline
\end{tabular}

Fonte: elaborado pelos autores.

Procedeu-se então preparação metalográfica com lixas de carbeto de silício com granulometria de 200 a 1200 mesh e polimento com alumina de $0,5 \mu \mathrm{m}$ e $1 \mu \mathrm{m}$.

A análise do teor de ferrita foi realizada segundo as normas ISO 8249:2000 utilizando o ferritoscópio Fisher FMP30, em que o aparelho tem uma faixa de medição entre 0,1 e 80\% de ferrita em padrões de calibragem. Foram tomadas medidas na superfície superior, inferior e na lateral das amostras, totalizando 9 medições em cada uma, antes e depois do envelhecimento.

A fim de comprovar o consumo da fase mais macia do aço duplex, foi ainda realizado ensaio de dureza Rockwell C com força de 187,5 Kgf e tempo de permanência de 30 segundos nas amostras. Cada amostra foi submetida a 5 indentações. 


\section{RESULTADOS E DISCUSSÕES}

A Tabela 2 exibe a diferença entre as frações volumétricas de ferrita antes e depois do tratamento térmico a $820^{\circ} \mathrm{C}$, onde $\Delta$ f é a diferença entre as frações finais e iniciais desta fase nas amostras.

Tabela 2: Variação do teor de ferrita antes e após envelhecimento.

\begin{tabular}{ccc}
\hline Amostra & Tempo [min] & $\Delta_{\mathrm{f}}[\mathrm{v}]$ \\
\hline A1 & 10 & $-8,633$ \\
\hline A2 & 30 & $-12,150$ \\
\hline A3 & 90 & $-19,956$ \\
\hline A4 & 120 & $-23,492$ \\
\hline A5 & 300 & $-34,031$ \\
\hline
\end{tabular}

Fonte: elaborado pelos autores.

Pode-se observar que a diferença aumenta com o tempo de tratamento térmico. Enquanto a amostra A1 submetida a um envelhecimento de 10 minutos apresentou uma redução de 8,6 na fração volumétrica de ferrita, a fração de ferrita consumida de forma mais significativa acontece na amostra A5, que teve um maior tempo de exposição.

A Figura 1 compara a fração de ferrita no material antes e após tratamento térmico.

Figura 1: Variação no teor de ferrita do aço.

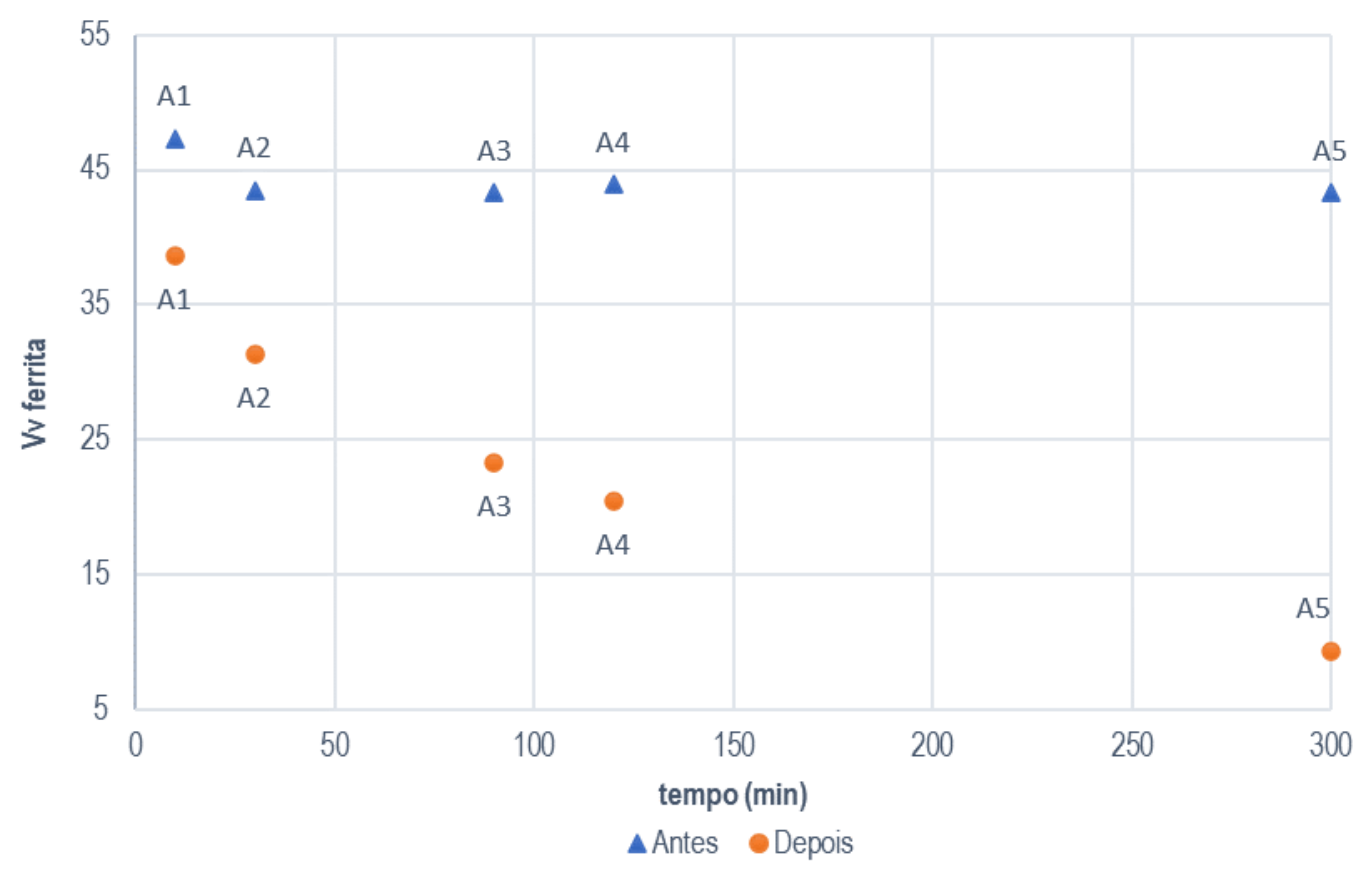


Fonte: elaborado pelos autores.

Nota-se que a fração volumétrica de ferrita decresce conforme o tempo de envelhecimento ao que o aço é exposto, onde acontece a consumição de ferrita em favor da nucleação e crescimento da fase sigma.

A Figura 2 exibe a variação da dureza com o envelhecimento.

Figura 2: Evolução da dureza Rockwell C do aço SAF2205.

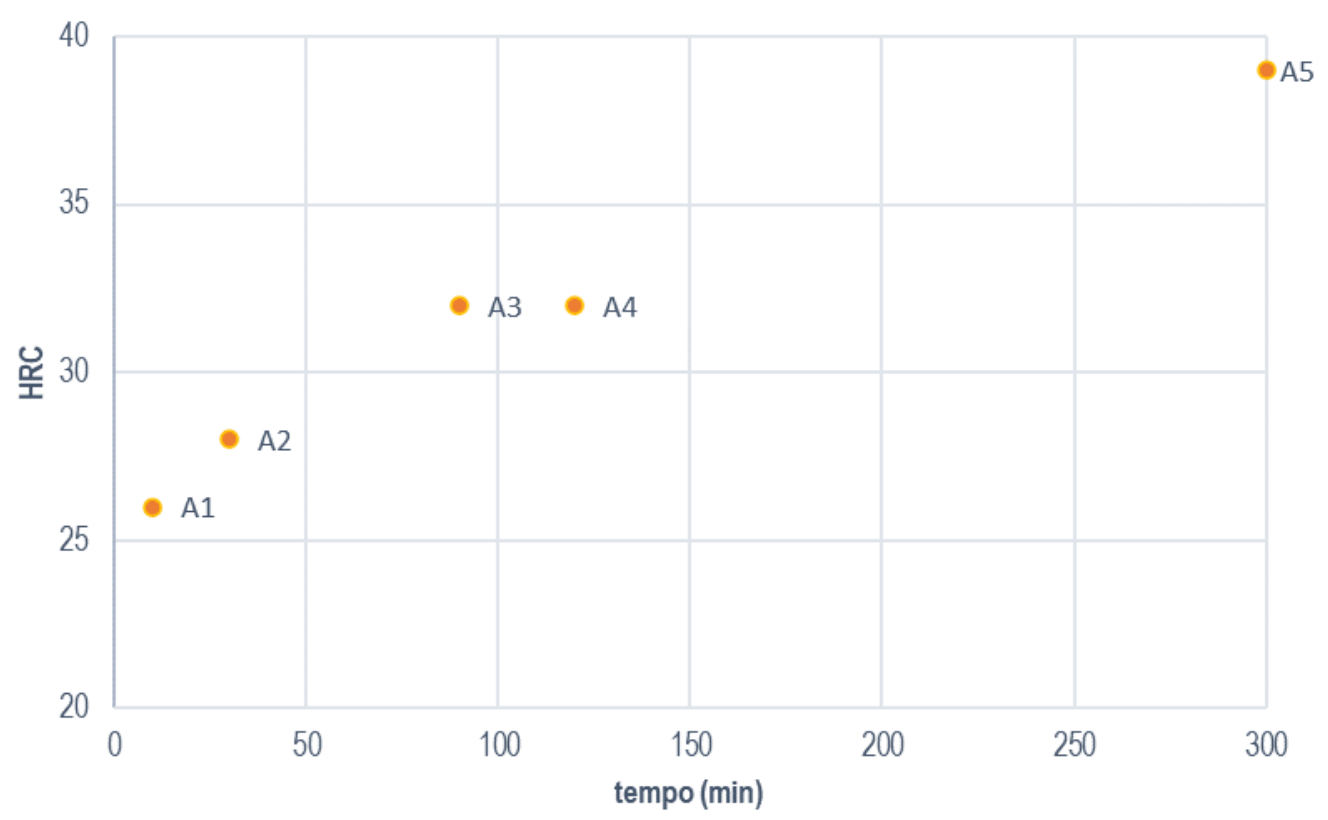

Fonte: elaborado pelos autores.

Ocorre o aumento da dureza do material conforme o tempo de tratamento. Pode-se associar este incremento na dureza em decorrência da nucleação da fase sigma, uma vez que o consumo de ferrita é mais severo na amostra A5 e menos severo na amostra A1.

\section{CONCLUSÃO}

A variável tempo no tratamento térmico a temperatura de $820{ }^{\circ} \mathrm{C}$ provocou uma degradação gradual da fração volumétrica de ferrita do aço, associado ao aumento da dureza do material. Essa mudança na microestrutura originalmente bifásica é relacionada a nucleação e o crescimento da fase sigma, visto que as condições de envelhecimento ao que aço foi exposto são favoráveis a cinética desta fase. 


\section{PRINCIPAIS REFERENCIAS}

CAMERINI, C. et al. Ferrite content meter analysis for delta ferrite evaluation in superduplex stainless steel. Journal of Materials Research and Technology, v. 7, n. 3, p. 366-370, 2018.

MENDONÇA, C. et al. Influência do tratamento térmico de envelhecimento a $850^{\circ} \mathrm{C}$ na microestrutura e nas propriedades mecânicas e magnéticas do aço Duplex UNS S31803. Matéria (Rio de Janeiro), v. 18, n. 3, p. 1373-1381, 2013.

OLIVEIRA, P.; BIGHETTI, W.; FONSECA, G. Estudo da formação da fase sigma em aço inoxidável superduplex UNS S32750 a 800 C. ABM Proceedings, 2015.

PADILHA, A. Materiais de engenharia. São Paulo (SP): HEMUS, 2007.

SANTOS, D. C. \& MAGNABOSCO, R. Estudo da corrosão seletiva em aço UNS S31803 (SAF 2205) em solução 1M HCl. 2008.

TAVARES, S. et al. Evaluation of Phases Proportions in Welded Joints of Duplex Stainless Steel by Ferritoscope and Metallographic Replica Analyses. [s.1.] IOS Press E-books, p. $290-301,2010$.

WANG, Y. et al. Pitting Corrosion of Thermally Aged Duplex Stainless Steels at Different Temperature for Long Time. Materials Research, v. 22, n. 6, 2019. 


\section{editoraomnisscientia@gmail.com M} https://editoraomnisscientia.com.br/

@editora_omnis_scientia @ https://www.facebook.com/omnis.scientia.9 f

$$
\text { +55 (87) 9656-3565 @ }
$$




\section{editoraomnisscientia@gmail.com M} https://editoraomnisscientia.com.br/ $\oplus$

@editora_omnis_scientia@ https://www.facebook.com/omnis.scientia.9 f

$$
\text { +55 (87) 9656-3565 @ }
$$

